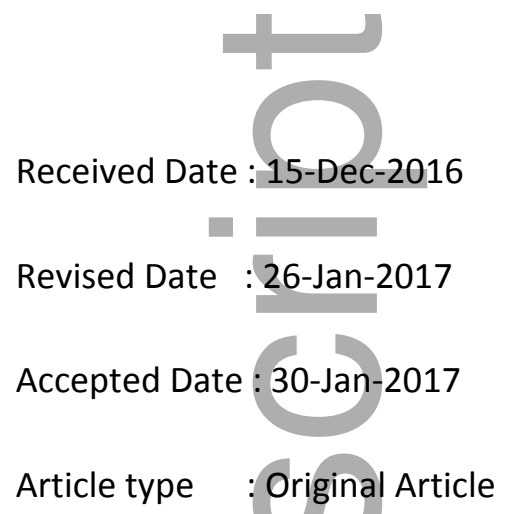

\title{
Small vessel disease and clinical outcomes after IV rt-PA treatment.
}

Francesco Arba, MD ${ }^{1,2}$; Domenico Inzitari, $\mathrm{MD}^{1}$; Myzoon Ali, $\mathrm{PhD}^{2}$; Steven J. Warach, MD, $\mathrm{PhD}^{3}$; Marie Luby, $\mathrm{PhD}^{4}$; Kennedy R. Lees MD, $\mathrm{FRCP}^{5}$; On behalf of the STIR/VISTA Imaging Collaboration*.

1. NEUROFARBA Department, University of Florence, Florence, Italy

2. Institute of Cardiovascular and Medical Sciences, Queen Elizabeth University Hospital Glasgow, Glasgow, United Kingdom

3. Department of Neurology, Dell Medical School, University of Texas at Austin, Austin, TX, USA

4. National Institute of Neurological Disorders and Stroke (NINDS), National Institutes of Health (NIH), Bethesda, MD, USA

5. Institute of Cardiovascular and Medical Sciences, University of Glasgow, C249 BHF Building, Glasgow, United Kingdom

This is the author manuscript accepted for publication and has undergone full peer review but has not been through the copyediting, typesetting, pagination and proofreading process, which may lead to differences between this version and the Version of Record. Please cite this article as doi: $\underline{10.1111 / a n e .12745}$

This article is protected by copyright. All rights reserved 
Word count: manuscript (excluding abstract, references, tables and figures) $=2513$; whole manuscript $=4177$

Key words: stroke, small vessel disease, white matter changes, magnetic resonance, intravenous thrombolysis, clinical outcomes

Running title: SVD and outcomes in intravenous thrombolysis

Corresponding author: Francesco Arba, $\mathrm{MD}, \mathrm{PhD}$

Email: $\underline{\text { francesco.arba@unifi.it }}$

Phone number: +39 055-7945518

Fax number: +39 055-7947665

\begin{abstract}
Introduction: Cerebral small vessel disease (SVD) contributes to dementia and disability in the elderly, and may negatively affect stroke outcomes. We aimed to evaluate to what extent single features and global burden of SVD detected with magnetic resonance (MR) are associated with worse outcomes in patients with ischaemic stroke treated with intravenous thrombolysis.
\end{abstract}

Methods: We accessed anonymised data and MR images from the Stroke Imaging Repository (STIR) and the Virtual International Stroke Trials Archive (VISTA) - Imaging. We described SVD features using validated scales and quantified the global burden of SVD with a combined score. Our main outcome was the modified Rankin Scale (mRS) at 90 days after stroke. We used logistic regression and ordinal regression models (adjusted for age, sex, stroke severity, onset to treatment time) to examine the associations between each SVD feature, SVD global burden and clinical outcomes.

Results: A total of 259 patients had MR scans available at baseline (mean age $\pm \mathrm{SD}=68.7 \pm 15.5$ years; 131 [49\%] males). After adjustment for confounders, severe white matter changes were associated with disability $(\mathrm{OR}=5.14 ; 95 \% \mathrm{CI}=2.30-11.48)$, functional dependency $(\mathrm{OR}=4.38 ; 95 \% \mathrm{CI}=2.10-9.13)$, and worse outcomes in ordinal analysis $(\mathrm{OR}=2.71 ; 95 \% \mathrm{CI}=1.25$ 5.85). SVD score was associated with disability $(\mathrm{OR}=1.66 ; 95 \% \mathrm{CI}=1.03-2.66)$ and functional dependency $(\mathrm{OR}=1.47 ; 95 \% \mathrm{CI}=1.00-2.45)$. Lacunes, enlarged perivascular spaces and brain atrophy showed no association with clinical outcomes.

This article is protected by copyright. All rights reserved 
Conclusion: our results suggest that SVD negatively affects stroke outcomes after intravenous thrombolysis. Although white matter changes seem to be the major driver in relation to worse outcomes, global estimation of SVD is feasible and may provide helpful information.

\section{Introduction}

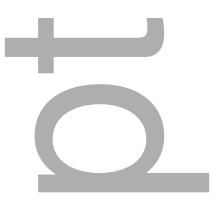

Cerebral small vessel disease (SVD) refers to various pathological processes that affect microvasculature in the brain. The most common type of SVD is age/hypertension related SVD, widely recognized as a risk factor for stroke ${ }^{1}$ and as the cause of lacunar strokes, which account for around $25 \%$ of all ischaemic strokes ${ }^{2}$. Imaging hallmarks of SVD can be detected with both computer tomography (CT) and magnetic resonance (MR). Although useful information can be gathered from CT scan, routinely used in clinical practice for assessment of acute ischaemic stroke patients suitable for recombinant tissue-Plasminogen Activator (rt-PA) treatment, MR is the gold standard imaging for visualization in vivo of SVD. MR imaging phenotype of SVD ranges from white matter changes (WMCs) to enlarged perivascular spaces (EPVS) ${ }^{3}$, and MR is the first choice for imaging in non-acute stroke setting.

A growing body of evidence, mostly from CT-based studies, focusses on the effect of a single feature of SVD (e.g. leukoaraiosis) on acute stroke treatment. Leukoaraiosis, the most extensively studied SVD feature in relation to acute ischaemic stroke and rt-PA treatment, and has been related to an increased risk of symptomatic intracerebral haemorrhagic transformation ${ }^{4,5,6}$ and worse clinical outcomes ${ }^{7,8,9}$. However, SVD is a heterogeneous process with a various imaging phenotype, and little is known about whether combinations of pre-existing imaging features can better convey predictive information on relevant outcomes. Staals et al. recently proposed a score ${ }^{8,9}$ to assess the global burden of SVD, introducing the concept that the whole spectrum of modifications rather than the single feature of SVD may provide valuable information for patients. Their score has been tested in a non-acute stroke scenario with MR assessment of SVD.

The global assessment of SVD burden among acute stroke patients has been only partly tested in one study. In a cohort of patients treated with rt-PA, leukoaraiosis did not affect subsequent functional outcome, and only lacunes were associated with death ${ }^{4}$. However, a re-analysis of the same dataset considering leukoaraiosis and lacunes as a combined score showed an independent association with both poor functional outcomes and symptomatic intracerebral hemorrhage after rt$\mathrm{PA}^{7}$.

This article is protected by copyright. All rights reserved 
In the present study, we aimed to explore the relationship between single and combined imaging findings of SVD and clinical outcomes in a cohort of patients with ischaemic stroke and available MR imaging before rt-PA treatment.

\section{Methods}

We accessed patient level data from the STroke Image Repository (STIR)|Virtual International Stroke Trials Archive (VISTA) Imaging consortium (http://stir.tacc.utexas.edu) and conducted a retrospective analysis. STIR/VISTA-Imaging consortium has been established to promote excellence in stroke care and stroke trial design. The overall purpose is to create an international consortium of investigators and a repository of source MRI and CT images to promote standardization and validation of acquisition, analytic, and clinical research methods of imagebased stroke research.

In the present study, we included patients with ischaemic stroke treated with intravenous (IV) rt-PA and accompanying MR based imaging before thrombolytic treatment. Additional ethical approval was not required since data were fully anonymous, from studies that had original ethical approval. Similarly, informed consent was not sought for the present study because data were anonymous. Clinical variables of interest included age, sex, baseline stroke severity assessed with National Institutes of Health Stroke Scale (NIHSS), onset to treatment time (OTT), baseline glucose, baseline blood pressure and cardiovascular risk factors. A stroke neurologist (FA), trained in MR assessment and blinded to clinical data, rated all the available scans (T1, T2, FLAIR sequences) for presence and severity of SVD features, according to STRIVE (STandards for ReportIng Vascular changes on nEuroimaging) recommendations ${ }^{2}$. We defined lacunes as round shaped cerebrospinal fluid isointense lesions measuring $\leq 20 \mathrm{~mm}$ in diameter on axial section in the white matter, basal ganglia or brainstem on T1, T2 or FLAIR sequences. We graded WMCs as 0-4 according to Van Swieten Scale (VSS) in anterior and posterior periventricular white matter ${ }^{13}$. Brain atrophy was defined as deep and cortical, and rated as none, mild-moderate and severe against a reference MR brain template $^{14}$. EPVS were defined as $\leq 2 \mathrm{~mm}$ round or linear cerebrospinal fluid isointense lesions (T2 hyperintense and T1/FLAIR hypointense). We separately rated EPVS in basal ganglia and centrum semiovale using a 5-point ordinal scale ${ }^{15}$ as follows: $0=$ no EPVS, 1=1-10 EPVS, $2=11$ to 20 , EPVS, $3=21$ to 40 EPVS, and 4= >40 EPVS.

To generate a global estimate of SVD, we built up an aggregate SVD score. Although previous versions of the SVD score included also microbleeds and EPVS ${ }^{19}$, we only considered MR imaging features of SVD detectable with CT scan, to allow transferability of results on clinical practice. We therefore combined the scores of white matter changes, lacunes, and brain atrophy. We assigned 1 This article is protected by copyright. All rights reserved 
point for each of the following: severe WMCs (VSS >=3), lacunes $>=2$ (multilacunar state) and severe brain atrophy, either central or cortical $(>=2)$. The combined four-points ordinal score assessed the global burden of SVD from 0 (no imaging features of severe SVD) to 3 (imaging features of SVD scored as severe for each imaging variable).

Our main outcome was the modified Rankin Scale (mRS) recorded 90 days after the index stroke. We considered mRS as ordinal scale (0-1=excellent outcome, 2-3=good outcome, 4-5=bad outcome, $6=$ death) and as dichotomous outcome (0-1 vs 2-6=disability/death; 0-2 vs 3-6=functional dependence/death; $6=$ death within three months).

Statistical analysis: We described general characteristics of the study population using basic descriptive statistics as appropriate. Our explanatory variables were each imaging feature of SVD and the SVD score. We run univariate analysis and retain variables with $\mathrm{p}<0.1$ in multivariate models. To assess independent associations, we adjusted univariate analysis for age, sex, NIHSS, OTT in both logistic and ordinal regression analysis. We considered $\mathrm{p}$ value $<0.05$ as statistically significant. Statistical analysis was carried out using SPSS for Windows (version 22.0; SPSS, Armonk NY, IBM Corp.).

\section{Results}

A total of 269 patients had available baseline MR scans. Ten scans were excluded for poor quality (movement artifacts and absence of relevant sequences), therefore 259 patients had scans available to assess white matter changes, brain atrophy and lacunes, whereas 164 patients had available T2 axial-section to assess EPVS. Baseline characteristics of study population are showed in Table 1. Mean age was $68.7 \pm 15.5$ years and 131 patients (49\%) were male. Baseline median NIHSS was 10 (IQR=6-17). Patients were treated with i.v. rt-PA with a mean of almost three hours after symptoms onset. Regarding SVD features, 92 (36\%) of patients had severe WMCs, 49 (19\%) had two or more lacunes, and $106(41 \%)$ had severe brain atrophy. High number of EPVS (grade 2-4) were more common in the centrum semiovale (142 patients, 87\%) than in the basal ganglia (92 patients, 56\%). Figure 1 shows distributions of single SVD features according to their grade. A total of 117 (45\%) patients had a SVD score of 0 , showing no signs of either white matter changes, lacunes or brain atrophy. Around a half of the whole population had some grade of a SVD, namely a score of 1 or 2 $(\mathrm{n}=118,45 \%)$, and 23 patients $(10 \%)$ had either severe white matter changes, two or more lacunes, and severe brain atrophy and scored SVD=3. Patients with increasing SVD score were older $(\mathrm{p}<0.001)$, were more likely prone to have hypertension and atrial fibrillation (Table 1).

Outcome three months after the index stroke was available for $143(55 \%)$ patients. Patients with

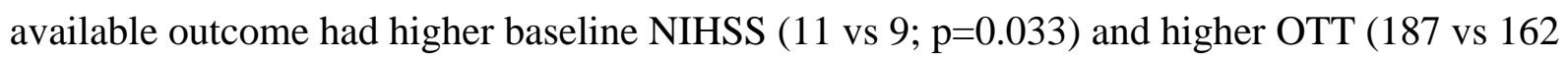
This article is protected by copyright. All rights reserved 
minutes; $\mathrm{p}=0.002$ ). There were no other differences with regard to relevant clinical variables (Supplemental Table 1). After three months from the index stroke, $90(60 \%)$ patients had a mRS=26 (disability/death); 77 (51\%) patients had a mRS=3-6 (functional dependency/death); 41 patients $(27 \%)$ died $(\mathrm{mRS}=6)$. Table 2 shows univariate and multivariate associations of SVD features and clinical outcomes. Multivariate logistic regression analysis confirmed the association between severe white matter changes and both disability/death $(\mathrm{OR}=4.74 ; 95 \% \mathrm{CI}=1.69-13.29)$ and functional dependence/death $(\mathrm{OR}=3.62 ; 95 \% \mathrm{CI}=1.38-9.50)$. Basal ganglia EPVS were associated with disability/death in univariate analysis $(\mathrm{OR}=2.47$; $95 \% \mathrm{CI}=1.13-5.43)$, however this association was confounded mainly by age in multivariate analysis $(\mathrm{OR}=1.84 ; 95 \% \mathrm{CI}=0.66-5.12)$. Neither lacunes nor brain atrophy were associated with clinical outcomes. SVD score was independently associated with both disability/death ( $\mathrm{OR}=1.66$ per SVD score point increase; 95\% CI=1.03-2.66) and functional dependence/death $(\mathrm{OR}=1.47$ per SVD score point increase; $95 \% \mathrm{CI}=1.00-2.45$; $\mathrm{p}=0.050$ ). None of the SVD radiological features showed association with death. After adjustment for age, sex, NIHSS and OTT, ordinal analysis showed that presence of severe white matter changes was independently associated with a shift towards worse outcomes $(\mathrm{OR}=2.71$; 95\% $\mathrm{CI}=1.25-5.85)$. Although not statistically significant, we found the same direction of association in patients with moderate-severe (grade 2 and 3) SVD score ( $\mathrm{OR}=1.94 ; 95 \% \mathrm{CI}=0.92-4.09 ; \mathrm{p}=0.087$ ) (Figure 2).

\section{Discussion}

We found that in a small cohort of ischaemic stroke patients treated with IV rt-PA, severe preexisting WMCs were associated with increased odds of poor clinical outcome. Global burden of SVD quantified with a combined score was also associated with disability and functional dependency. Lacunes, brain atrophy and EPVS showed no association with relevant clinical outcomes.

Our findings regarding WMCs are in keeping with previous larger studies that showed a negative association between WMCs grade and clinical outcomes ${ }^{8,9}$. We built on these studies by applying the gold standard tool for detection of SVD features ${ }^{3}$ (i.e. MR) and confirmed the effect of WMCs on outcome on a smaller dataset. As a common imaging marker of SVD, WMCs could affect stroke outcomes through various mechanisms. In the hyperacute phase of ischaemic stroke, WMCs may influence the infarct volume and are associated with a larger growth of the infarct size ${ }^{16,17,18}$. WMCs are also associated with larger cortical infarcts after distal arterial occlusion ${ }^{19}$, suggesting that microcirculation could be affected also in the grey matter and therefore lead to greater cerebral damage. Patients with WMCs display reduced vascular density ${ }^{20}$, impaired cerebral perfusion ${ }^{21}$ and SVD is related to both endothelial dysfunction and blood brain barrier leakage, which are thought to This article is protected by copyright. All rights reserved 
be key drivers of its pathophysiology $\mathrm{y}^{22,23,24}$. Furthermore, rheological modifications such as increased platelet activation ${ }^{25}$ and a pro-coagulative status may occur together with $\mathrm{SVD}^{26}$. Each of the aforementioned physiopathological processes related to SVD may eventually result in a larger infarct size, impaired reperfusion after recanalization therapy and, from a clinical point of view, in worse outcomes after stroke.

Although our study sample was smaller than previous studies ${ }^{8,9}$, we found statistically significant associations. Compared to plain CT scan, MR is more sensitive in detection of WMCs ${ }^{13,27}$, hence it is reasonable that larger sample sizes are required to reach significant results when using CT, particularly regarding the evaluation of WMCs. On the other hand, agreement of visual rating scales for WMCs between MR and CT is fairly $\operatorname{good}^{28}$, and both WMCs and brain atrophy scales have been validated for $\mathrm{MR}$ and $\mathrm{CT}^{8,13}$. Conversely to a previous larger study ${ }^{4}$, we did not found any independent association between lacunes and clinical outcomes. The sample size and the consequent lack of adequate statistical power may be the reason for this discrepancy.

We also investigated EPVS in relation to acute stroke outcomes. EPVS are an imaging hallmark of SVD and have been associated with cognitive decline after stroke ${ }^{29,30}$. We found EPVS common in patients with stroke, and their relation with clinical outcomes may be of clinical interest. We found a significant association with poor outcomes in the univariate analysis; however our study was likely underpowered to demonstrate an independent association after multivariable adjustment. Future studies may specifically address whether EPVS have a predictive role in stroke outcome and in patients treated with rt-PA or mechanical thrombectomy.

SVD features frequently occur together, and we tried to quantify the cumulative effect of combined signs. Considerably, we found signs of two or more severe SVD features in around one third of our patients, and this negatively affected clinical outcomes. Therefore, we showed that quantification of global burden of SVD is feasible and has clinical relevance, as previously suggested by other groups ${ }^{10,11}$. We did not assess the entire imaging phenotype of SVD detectable with MR, rather we chose features visible with both MR and plain CT scan to enhance the feasibility of use in clinical practice. We observed a meaningful association between the SVD score and clinical outcomes, with WMCs acting as the driving feature for poor outcome. We acknowledge that the SVD score requires further validation with larger sample size in acute stroke setting and with CT scan evaluation of SVD features. However, if validated, the score might be applied in both clinical practice and clinical trials and increase the power to predict outcomes. Moreover, in an acute stroke scenario, the use of the SVD score may redress CT limitations in the detection of single SVD features and provide more accurate estimates about stroke prognosis. 
The strengths of our study include the blinded rating of SVD features, the use of the gold standard for SVD assessment in vivo (i.e. MR imaging), and the qualitative rating of SVD features with extensively validated scales, easily transferrable to clinical practice. The small sample size is the main limitation of our study, and we recognize that our results related to the SVD score need external validation in larger cohorts. Another limit is the number (almost a half) of patients with missed outcomes at follow-up. Assuming that missed patients generally face worse outcomes compared to non-missed patients, one could argue that our patients with available outcomes had a milder stroke profile. However, the comparison of baseline characteristics of included and excluded patients does not seem to support this attrition bias. In fact, stroke severity and OTT, two of the most important predictors of unfavourable outcomes, were higher (i.e. increased odds of poor outcome) in the population with available outcomes at follow-up.

In conclusion, we showed that in a cohort of MR-based imaging patients with acute ischaemic stroke treated with IV rt-PA, WMCs are frequent and associated with disability, functional dependency and a shift towards worse outcomes in ordinal analysis. We also utilized a combined score that takes into account pre-existing global burden of SVD radiological features and found the score associated with disability and functional dependency. The SVD score is potentially feasible for application in an acute stroke setting, and after validation for CT scan could inform the risk of attaining a poorer outcome after rt-PA treatment, particularly in patients with two or more signs of severe SVD; this may also inform the design of future clinical trials. Our results lend support to the theory that SVD may act as a marker of brain frailty; the presence of SVD, and particularly WMCs, have negative effects on clinical outcomes.

\section{Disclosures: none.}

Ackowledgements: This work was also supported by the Department of Neurology, Dell Medical School, University of Texas at Austin, Austin, TX, USA and the National Institute of Neurological Disorders and Stroke (NINDS), and the National Institutes of Health (NIH), Bethesda, MD, USA. Francesco Arba received funding from Ente Cassa di Risparmio di Firenze (2010.06.03).

\section{*Appendix}

STIR/VISTA Imaging steering committee includes Gregory W. Albers, Stephen M. Davis, Geoffrey A. Donnan, Marc Fisher, Anthony J. Furlan, James C. Grotta, Werner Hacke, Dong-Wha This article is protected by copyright. All rights reserved 
Kang, Chelsea Kidwell, Walter J. Koroshetz, Kennedy R. Lees, Michael H. Lev, David S.

Liebeskind, A. Gregory Sorensen, Vincent N. Thijs, Götz Thomalla, Steven J. Warach, Joanna M. Wardlaw, and Max Wintermark.



\section{References:}

1. Inzitari D. Leukoaraiosis: an independent risk factor for stroke? Stroke. 2003; 34:2067-71.

2. Wardlaw JM, Smith C, Dichgans M. Mechanisms of sporadic cerebral small vessel disease: insights from neuroimaging. Lancet Neurol. 2013;12(5):483-97.

3. Wardlaw JM, Smith EE, Biessels GJ, et al.; STandards for ReportIng Vascular changes on nEuroimaging (STRIVE v1). Neuroimaging standards for research into small vessel disease and its contribution to ageing and neurodegeneration. Lancet Neurol. 2013; 12:822-38.

4. Palumbo V, Boulanger JM, Hill MD, Inzitari D, Buchan AM; CASES Investigators. Leukoaraiosis and intracerebral hemorrhage after thrombolysis in acute stroke. Neurology. 2007; 68:1020-1024.

5. Neumann-Haefelin T, Hoelig S, Berkefeld J, et al; MR Stroke Group. Leukoaraiosis is a risk factor for symptomatic intracerebral hemorrhage after thrombolysis for acute stroke. Stroke. 2006; 37: 2463-6.

6. Curtze S, Haapaniemi E, Melkas S, Mustanoja S, Putaala J, Sairanen T, Sibolt G, Tiainen M, Tatlisumak T, Strbian D. White Matter Lesions Double the Risk of Post-Thrombolytic Intracerebral Hemorrhage. Stroke. 2015; 46(8):2149-55.

7. Arba F, Palumbo V, Boulanger JM, Pracucci G, Inzitari D, Buchan AM, Hill MD; CASES Investigators. Leukoaraiosis and lacunes are associated with poor clinical outcomes in ischemic stroke patients treated with intravenous thrombolysis. Int J Stroke. 2016;11:62-7.

8. IST-3 collaborative group. Association between brain imaging signs, early and late outcomes, and response to intravenous alteplase after acute ischaemic stroke in the third International Stroke Trial (IST-3): secondary analysis of a randomised controlled trial. Lancet Neurol. 2015; 14:485-96.

This article is protected by copyright. All rights reserved 
9. Curtze S, Melkas S, Sibolt G, Haapaniemi E, Mustanoja S, Putaala J, Sairanen T, Tiainen M, Tatlisumak T, Strbian D. Cerebral computed tomography-graded white matter lesions are associated with worse outcome after thrombolysis in patients with stroke. Stroke. 2015; 46(6):1554-60.

10. Staals J, Makin SD, Doubal FN, Dennis MS, Wardlaw JM. Stroke subtype, vascular risk factors, and total MRI brain small-vessel disease burden. Neurology. 2014; 83:1228-34.

11. Staals J, Booth T, Morris Z, Bastin ME, Gow AJ, Corley J, et al. Total MRI load of cerebral small vessel disease and cognitive ability in older people. Neurobiol Aging. 2015. pii: S0197-4580(15)00335-8. doi: 10.1016/j.neurobiolaging. 2015.06.024.

12. Wardlaw JM, Smith EE, Biessels GJ, Cordonnier C, Fazekas F, Frayne R, et al.; STandards for ReportIng Vascular changes on nEuroimaging (STRIVE v1). Neuroimaging standards for research into small vessel disease and its contribution to ageing and neurodegeneration. Lancet Neurol. 2013; 12:822-38.

13. Van Swieten JC, Hijdra A, Koudstaal PJ, van Gijn J. Grading white matter lesions on CT and MRI: a simple scale. J Neurol Neurosurg Psychiatry. 1990; 53:1080-3.

14. Farrell C, Chappell F, Armitage PA, Keston P, Maclullich A, Shenkin S, Wardlaw JM. Development and initial testing of normal reference MR images for the brain at ages 65-70 and 75-80 years. Eur Radiol. 2009; 19(1):177-83.

15. Potter GM, Chappell FM, Morris Z, et al. Cerebral perivascular spaces visible on magnetic resonance imaging: development of a qualitative rating scale and its observer reliability. Cerebrovasc Dis. 2015; 39:224-31.

16. Henninger N, Lin E, Haussen DC, Lehman LL, Takhtani D, Selim M, Moonis M. Leukoaraiosis and sex predict the hyperacute ischemic core volume. Stroke. 2013; 44(1):617.

17. Ay H, Arsava EM, Rosand J, et al.Severity of leukoaraiosis and susceptibility to infarct growth in acute stroke. Stroke. 2008; 39(5):1409-13.

18. Helenius J, Mayasi Y, Henninger N. White matter hyperintensity lesion burden is associated with the infarct volume and 90-day outcome in small subcortical infarcts. Acta Neurol Scand. 2016. doi: 10.1111/ane.12670.

19. Henninger N, Khan MA, Zhang J, Moonis M, Goddeau RP Jr. Leukoaraiosis predicts cortical infarct volume after distal middle cerebral artery occlusion. Stroke. 2014; 45:689-95.

20. Moody DM, Thore CR, Anstrom JA, et al. Quantification of afferent vessels shows reduced brain vascular density in subjects with leukoaraiosis. Radiology. 2004; 233:883-890. 
21. Topakian R, Garrick TR, Howe FA, et al. Blood-brain barrier permeability is increased in normal-appearing white matter in patients with lacunar stroke and leukoaraiosis. J Neurol Neurosurg Psychiatry 2010; 81(2): 192-7.

22. Huynh TJ, Murphy B, Pettersen JA, Tu H, Sahlas DJ, Zhang L, et al. CT perfusion quantification of small-vessel ischemic severity. Am J Neuroradiol. 2008; 29:1831-6.

23. Wardlaw JM, Doubal F, Armitage P, Chappell F, Carpenter T, Muñoz Maniega S, et al. Lacunar stroke is associated with diffuse blood-brain barrier dysfunction. Ann Neurol. 2009; 65:194-202.

24. Huisa BN, Caprihan A, Thompson J, Prestopnik J, Qualls CR, Rosenberg GA. Long-term blood brain permeability changes in Binswanger disease. Stroke. 2015; 46:2413-8.

25. Iwamoto T, Kubo H, Takasaki M. Platelet activation in the cerebral circulation in different subtypes of ischaemic stroke and Binswanger's disease. Stroke. 1995; 26:52-6.

26. Tomimoto H, Akiguchi I, Wakita H, Osaki A, Hayashi M, Yamamoto Y. Coagulation activation in patients with Binswanger disease. Arch Neurol. 1999; 56:1104-8.

27. Wahlund LO, Barkhof F, Fazekas F, Bronge L, Augustin M, Sjögren M, et al; European Task Force on Age-Related White Matter Changes. A new rating scale for age-related white matter changes applicable to MRI. matter changes applicable to MRI and CT. Stroke. 2001; 32:1318-1322.

28. Wattjes MP, Henneman WJ, van der Flier WM, de Vries O, Träber F, Geurts JJ, Scheltens P, Vrenken H, Barkhof F. Diagnostic imaging of patients in a memory clinic: comparison of MR imaging and 64-detector row CT. Radiology. 2009; 253:174-83.

29. Huijts M, Duits A, Staals J, et al. Basal ganglia enlarged perivascular spaces are linked to cognitive function in patients with cerebral small vessel disease. Curr Neurovasc Res. 2014.

30. Arba F, Quinn TJ, Hankey GJ et al; VISTA Collaboration. Enlarged perivascular spaces and cognitive impairment after stroke and transient ischaemic attack. Int J Stroke. 2016. pii:1747493016666091.

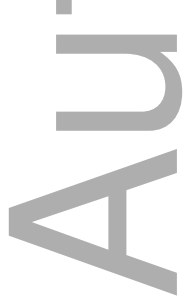




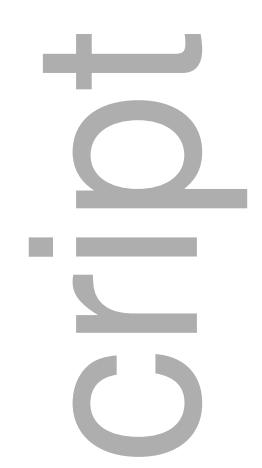

Table 1. Demographic and clinical characteristics of study population according to SVD score.

\begin{tabular}{|c|c|c|c|c|c|c|}
\hline 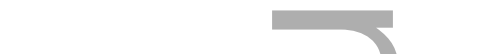 & & \multicolumn{4}{|c|}{ SVD score } & \multirow[b]{2}{*}{$\mathbf{p}$} \\
\hline Variables & $\begin{array}{c}\text { Total } \\
\text { population } \\
\mathrm{N}=259\end{array}$ & $\begin{array}{c}0 \\
(n=117)\end{array}$ & $\begin{array}{c}1 \\
(n=59)\end{array}$ & $\begin{array}{c}2 \\
(n=59)\end{array}$ & $\begin{array}{c}3 \\
(n=23)\end{array}$ & \\
\hline Age, mean $( \pm \mathrm{SD})$ & $69.7 \pm 15.5$ & $60.9 \pm 15.5$ & $72.4 \pm 13.2$ & $80.2 \pm 7.8$ & $80.5 \pm 7.6$ & $<0.001$ \\
\hline Sex, male & $131(49)$ & $61(52)$ & $27(46)$ & $28(48)$ & $10(48)$ & 0.791 \\
\hline Baseline NIHSS, median (IQR) & $10(6-17)$ & $9(5-15)$ & $10(6-19)$ & $13(8-19)$ & $11(7-15)$ & 0.047 \\
\hline OTTT, mean $( \pm$ SD) & $176.32 \pm 62.4$ & $171.4 \pm 60.8$ & $181.5 \pm 65.5$ & $175.6 \pm 63.8$ & $200.7 \pm 65.0$ & 0.245 \\
\hline Glucose, mean $( \pm S$ & $133.1 \pm 51.6$ & $132.5 \pm 52.9$ & $128.5 \pm 40.7$ & $130.7 \pm 38.3$ & $153.8 \pm 85.4$ & 0.226 \\
\hline Systolic BP, mean $( \pm$ SD $)$ & $152.2 \pm 26.2$ & $148.4 \pm 24.0$ & $154.2 \pm 29.6$ & $159.1 \pm 28.1$ & $152.0 \pm 23.5$ & 0.255 \\
\hline Diastolic BP, mean $( \pm \mathrm{SD})$ & $81.9 \pm 15.2$ & $79.3 \pm 13.2$ & $85.5 \pm 17.4$ & $84.3 \pm 16.1$ & $81.0 \pm 14.2$ & 0.130 \\
\hline Diabetes & $57(21)$ & $23(20)$ & $14(23)$ & $11(19)$ & $8(35)$ & 0.381 \\
\hline Hypertension & $185(69)$ & $68(58)$ & $50(85)$ & $45(76)$ & $19(83)$ & 0.001 \\
\hline Atrial fibrillation & 79 (29) & $22(19)$ & $20(34)$ & $26(44)$ & $10(44)$ & 0.002 \\
\hline Hyperlipidemia & $123(46)$ & $54(46)$ & $34(58)$ & $23(39)$ & $9(39)$ & 0.188 \\
\hline Smoke & $73(27)$ & $32(27)$ & $13(22)$ & 17 (29) & $7(30)$ & 0.806 \\
\hline
\end{tabular}


$\mathrm{SD}=$ Standard deviation; NIHSS=National Institutes of Health Stroke Scale; IQR=Interquartile Range; OTT=Onset To Treatment time; BP=Blood Pressure; TIA=Transient Ischaemic Attack. Data are numbers (\%) unless otherwise stated.

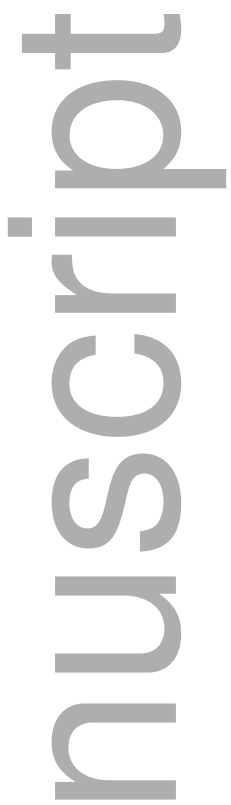

Table 2. Unadjusted and adjusted associations between radiological features of small vessel disease and clinical outcomes.

\begin{tabular}{|c|c|c|c|c|c|c|}
\hline \multirow[b]{2}{*}{ Variables } & \multicolumn{3}{|c|}{ Unadjusted OR (95\% CI) } & \multicolumn{3}{|c|}{ Adjusted OR (95\% CI) } \\
\hline & $m R S=2-6$ & $m R S=3-6$ & $\mathrm{mRS}=6$ & $m R S=2-6$ & $m R S=3-6$ & $\mathrm{mRS}=6$ \\
\hline Severe WMCs & $5.14(2.30-11.48)$ & $4.38(2.10-9.13)$ & $2.60(1.21-5.59)$ & $4.74(1.69-13.29)$ & $3.62(1.38-9.50)$ & $1.72(0.65-4.60)$ \\
\hline Two or more lacunes & $1.77(0.71-4.39)$ & $1.84(0.77-4.39)$ & $0.65(0.23-1.88)$ & - & - & - \\
\hline Basal ganglia EPVS & $2.47(1.13-5.43)$ & $1.72(0.80-3.68)$ & $2.38(0.95-5.95)$ & $1.84(0.66-5.12)$ & - & - \\
\hline Centrum semiovale EPVS & $1.36(0.46-3.96)$ & $1.18(0.41-3.41)$ & $1.11(0.33-3.77)$ & - & - & - \\
\hline Central brain atrophy & $1.65(1.06-2.57)$ & $1.52(0.98-2.36)$ & $1.17(0.71-1.93)$ & $0.94(0.48-1.83)$ & - & - \\
\hline Cortical brain atrophy & $1.49(0.87-2.57)$ & $1.54(0.90-2.62)$ & $1.11(0.61-2.02)$ & - & - & - \\
\hline SVD score & $1.93(1.32-2.81)$ & $1.88(1.31-2.69)$ & $1.25(0.87-1.81)$ & $1.66(1.03-2.66)$ & $1.47(1.00-2.45)$ & - \\
\hline
\end{tabular}

WMCs=White Matter Changes; EPVS=Enlarged Perivascular Spaces; SVD=Small Vessel Disease;

OR=Odds Ratio; $\mathrm{CI}=$ Confidence Interval; $\mathrm{mRS}=$ modified Rankin Scale. 
Figure 1. Distribution of SVD features according to their severity.


SVD=Small Vessel Disease; WMCs=White Matter Changes; EPVS=Enlarged Perivascular Spaces. N for WMCs and brain atrophy=259; N for lacunes=258; $\mathrm{N}$ for EPVS=164. This article is protected by copyright. All rights reserved 
Figure 2. Crude rates of effect of severe white matter changes (WMCs) and SVD score on cathegorized mRS. Odds ratios are from ordinal regression analysis adjusted for age, sex, baseline NIHSS, onset to treatment time.

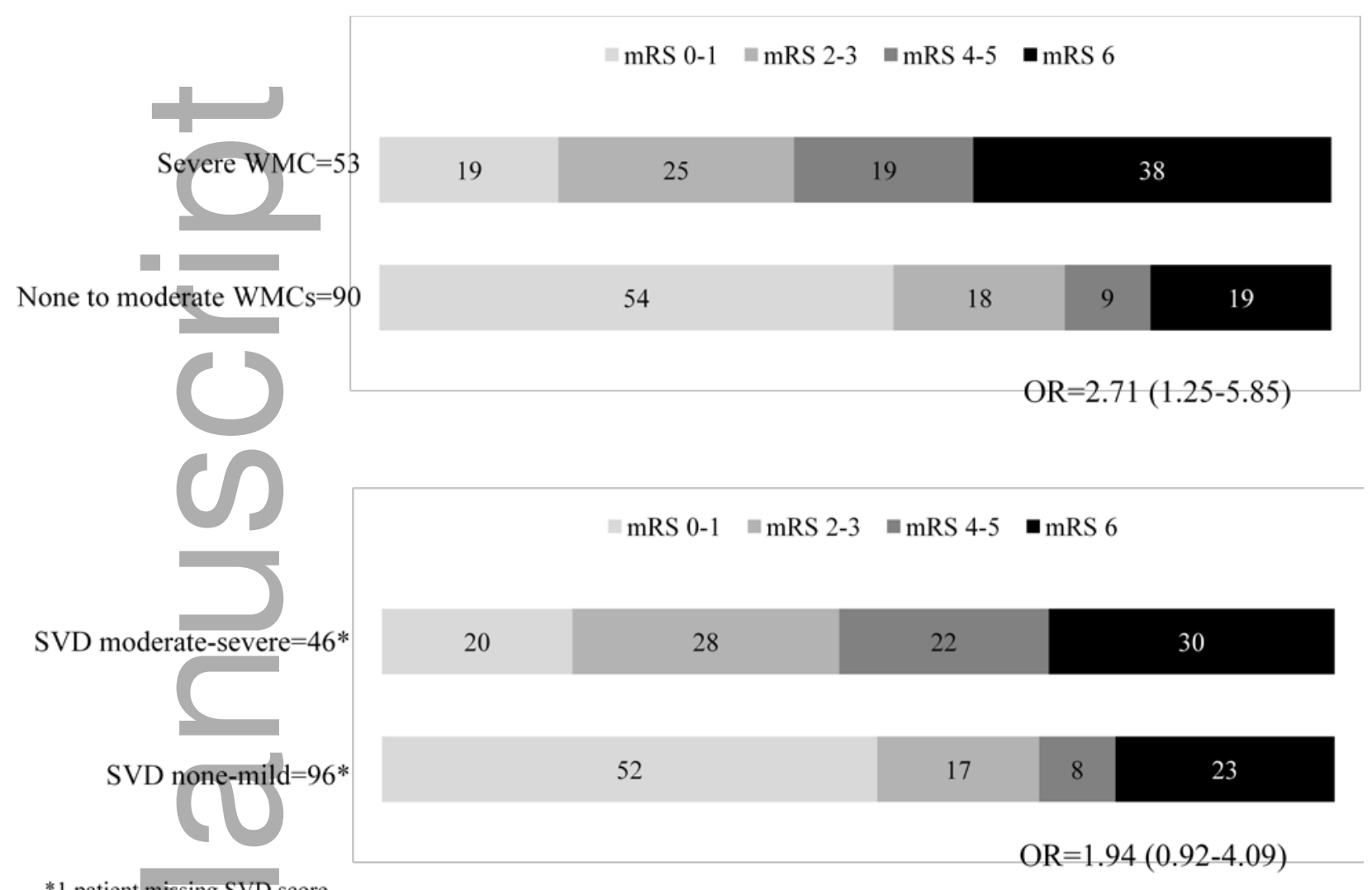

*1 patient missing SVD score

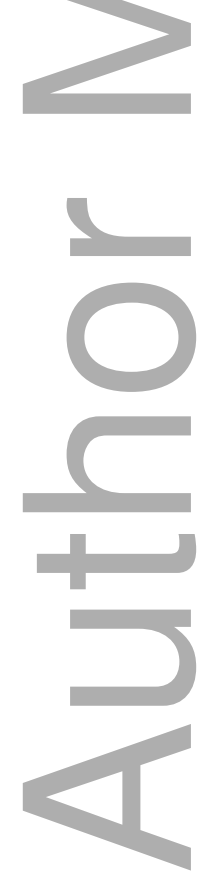

This article is protected by copyright. All rights reserved 


\section{University Library}

\section{- M M I N E R VA A gateway to Melbourne's research publications}

Minerva Access is the Institutional Repository of The University of Melbourne

Author/s:

Arba, F;Inzitari, D;Ali, M;Warach, SJ;Luby, M;Lees, KR

Title:

Small vessel disease and clinical outcomes after IV rt-PA treatment

Date:

2017-07-01

Citation:

Arba, F., Inzitari, D., Ali, M., Warach, S. J., Luby, M. \& Lees, K. R. (2017). Small vessel disease and clinical outcomes after IV rt-PA treatment. ACTA NEUROLOGICA SCANDINAVICA, 136 (1), pp.72-77. https://doi.org/10.1111/ane.12745.

Persistent Link:

http://hdl.handle.net/11343/292483 